PROCEEDINGS OF THE

AMERICAN MATHEMATICAL SOCIETY

Volume 130, Number 7, Pages 1927-1936

S 0002-9939(01)06298-0

Article electronically published on December 31, 2001

\title{
ON REPRESENTABLE LINEARLY COMPACT MODULES
}

\author{
NGUYEN TU CUONG AND LE THANH NHAN
}

(Communicated by Wolmer V. Vasconcelos)

\begin{abstract}
For a flat $R$-module $F$, we prove that $\operatorname{Hom}_{R}(F,-)$ is a functor from the category of linearly compact $R$-modules to itself and is exact. Moreover, $\operatorname{Hom}_{R}(F, M)$ is representable when $M$ is linearly compact and representable. This gives an affirmative answer to a question of L. Melkersson (1995) for linearly compact modules without the condition of finite Goldie dimension. The set of attached prime ideals of the co-localization $\operatorname{Hom}_{R}\left(R_{S}, M\right)$ of a linearly compact representable $R$-module $M$ with respect to a multiplicative set $S$ in $R$ is described.
\end{abstract}

\section{$\S 1$. INTRODUCTION}

Let $R$ be a commutative ring and $M$ an $R$-module. L. Melkersson and P. Schenzel in [M-S] called the module $\operatorname{Hom}_{R}\left(R_{S}, M\right)$ the co-localization of $M$ with respect to a multiplicative set $S$ in $R$. They showed in this paper that their construction has many interesting properties when $M$ is an Artinian module. For example, the co-localization of $M$ has a secondary representation in the sense of I. G. Macdonald M2 and the functor $\operatorname{Hom}_{R}\left(R_{S},-\right)$ is then an exact functor from the category of Artinian $R$-modules to the category of $R$-modules. However, they also proved that this functor is not closed on the category of Artinian $R$-modules. Namely, the co-localization of an Artinian module does not usually give an Artinian module, and it may even have infinite Goldie dimension. The purpose of this paper is to extend the main results of Melkersson-Schenzel for Artinian modules presented in [M-S] to the class of all linearly compact representable $R$-modules. This class strictly contains all Artinian $R$-modules. Moreover, instead of the functor of colocalization, we consider the functor $\operatorname{Hom}_{R}(F,-)$ with $F$ a flat module. Then we show that this functor is closed with respect to the class of all linearly compact representable modules. Therefore we get a positive answer to an open question of Melkersson [Me $\S 5]$ in the case that $M$ is a linearly compact module without the condition of finite Goldie dimension. It should be mentioned that the concept of linear compactness was first introduced by S. Lefschetz [Lef] for vector spaces of arbitrary dimension and extended for modules by D. Zelinsky [Z, H. Leptin [Lep1, Lep2] and it plays an important role for duality in algebra.

Received by the editors September 20, 2000 and, in revised form, February 1, 2001.

1991 Mathematics Subject Classification. Primary 13C05; Secondary 13J99.

Key words and phrases. Linearly compact module, secondary representation, co-localization.

This work was supported in part by the National Basis Research Program in Natural Science of Vietnam.

(C)2001 American Mathematical Society 
This paper is divided into 4 sections. In the next section, we recall some basic facts about linearly compact modules following [M1, [Z]. If $M$ is a linearly compact module and $F$ a flat $R$-module, we show in this section that $\operatorname{Hom}_{R}(F, M)$ can carry a linear topology which is derived from a direct system $\left\{F_{t}\right\}$ of free $R$-modules of finite type such that $F=\underset{t \in K}{\lim _{t \in}} F_{t}$. This topology is linearly compact and independent of the choice of the direct system $\left\{F_{t}\right\}$, therefore $\operatorname{Ext}_{R}^{i}(F, M)=0$ for all $i>0$ (Theorem 2.4). It follows as an immediate consequence that the functor $\operatorname{Hom}_{R}(F,-)$ is exact on the category of linearly compact $R$-modules to itself. We suppose in addition in $\S 3$ that $M$ is representable. The main result of this section is then to show that $\operatorname{Hom}_{R}(F, M)$ is also representable (Theorem 3.6). This result gives an affirmative answer to a question of $\mathrm{L}$. Melkersson "is the module $\operatorname{Hom}_{R}(F, M)$ representable when $M$ is a representable $R$-module of finite Goldie dimension and $F$ is a flat $R$-module?" (see [Me, $\S 5]$ ) for a linearly compact module without the assumption that $M$ is of finite Goldie dimension. Starting from a minimal secondary representation of $M$ we can construct in $\S 4$ a minimal secondary representation of the co-localization $\operatorname{Hom}_{R}\left(R_{S}, M\right)$ by a method similar to that used in [M-S]. This will give a description of the set of attached prime ideals of $\operatorname{Hom}_{R}\left(R_{S}, M\right)$. The technique of co-localization is then applied to describe the attached prime ideals of a tensor product $N \otimes M$, where $N$ is a finitely generated $R$-module and $M$ is a linearly compact representable $R$-module.

\section{§2. Linearly compact modules}

Throughout this paper, let $R$ be a commutative topological ring and $M$ a topological $R$-module. A nucleus of $M$ is a neighborhood of the zero element of $M$ and a nuclear base of $M$ is a base for the nuclei of $M$.

First we recall the concept of linearly compact modules by using the terminology of I. G. Macdonald [M1].

Definition 2.1. (i) $M$ is said to be linearly topologized if $M$ has a nuclear base $\mathcal{M}$ consisting of open submodules.

(ii) A Hausdorff linearly topologized $R$-module $M$ is said to be linearly compact if $M$ has the following property: if $\mathcal{F}$ is a family of closed cosets (i.e. the cosets of closed submodules) in $M$ which has the finite intersection property, then the cosets in $\mathcal{F}$ have a non-empty intersection.

Now we present some facts about linearly compact modules which are often used in this paper.

Lemma 2.2 (See $[\mathrm{M} 1$ and $[\mathrm{Z}]$ ). (i) Let $M$ be $a$ linearly compact $R$-module and $N$ a submodule of $M$. Then $N$ is closed if and only if $N$ is linearly compact.

(ii) If $M, N$ are Hausdorff linearly topologized $R$-modules, $M$ is linearly compact and $f: M \longrightarrow N$ is a continuous homomorphism, then $f(M)$ is linearly compact and hence $f$ is a closed map.

(iii) If $M$ is a Hausdorff linearly topologized $R$-module and $N$ is a closed submodule of $M$, then $M$ is linearly compact if and only if $N$ and $M / N$ are linearly compact.

(iv) The direct product of linearly compact $R$-modules is linearly compact where the topology is the product topology.

(v) The inverse limit of an inverse system of linearly compact $R$-modules and continuous homomorphisms is linearly compact with the obvious topology. 
(vi) If $M$ is a Hausdorff linearly topologized $R$-module and $N_{1}, \ldots, N_{r}$ are linearly compact submodules of $M$, then $N_{1}+\ldots+N_{r}$ is linearly compact with the induced topology.

Lemma 2.3 (See J, Theorem 7.1]). Let $\left\{M_{t}\right\}$ be inverse system of linearly compact $R$-modules and continuous homomorphisms. Then $\underbrace{\lim ^{(i)}}_{t}\left(M_{t}\right)=0$ for all $i>0$, where $\underbrace{\lim ^{(i)}}_{t}(-)$ is the $i-$ th right derived functor of the inverse limit.

Let $P$ be a free $R$-module with a base $\left\{x_{i}\right\}_{i \in I}$ and $M$ a linearly topologized $R$-module. According to C. U. Jensen [J, Theorem 7.4] we can define the topology on the module $\operatorname{Hom}_{R}(P ; M)$ as the product topology $M^{I}$ via the isomorphism $\operatorname{Hom}_{R}(P ; M) \cong M^{I}$. Moreover, if $f: P \longrightarrow P^{\prime}$ is a homomorphism of two free $R$-modules, the induced homomorphism $f_{*}: \operatorname{Hom}_{R}\left(P^{\prime} ; M\right) \longrightarrow \operatorname{Hom}_{R}(P ; M)$ is continuous.

Now, let $F$ be a flat $R$-module. There exists by Lazard $[\mathrm{L}]$ a direct system $\left\{F_{t}\right\}$ of free $R$-modules of finite type such that $F \cong \underset{t}{\lim } F_{t}$. Hence $\left\{\operatorname{Hom}_{R}\left(F_{t}, M\right)\right\}$ forms an inverse system of linearly topologized $R$-modules with continuous ho-

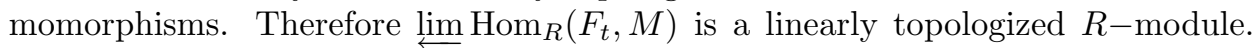
Thus we define the topology of $\operatorname{Hom}_{R}(F, M)$ by the topology of the inverse limit $\underset{l i m}{\longleftarrow} \operatorname{Hom}_{R}\left(F_{t}, M\right)$ and the isomorphisms

$$
\operatorname{Hom}_{R}(F, M) \cong \operatorname{Hom}_{R}\left(\underset{t}{\lim } F_{t}, M\right) \cong \underbrace{\lim }_{t} \operatorname{Hom}_{R}\left(F_{t}, M\right) .
$$

In this case we say that the topology of $\operatorname{Hom}_{R}(F, M)$ is defined by the direct system $\left\{F_{t}\right\}$.

It should be mentioned that in general there exist inverse systems of linearly topologized modules with continuous homomorphisms whose inverse limits are algebraically isomorphic but such that their topologies, which are determined from these inverse systems as above, are not equivalent. For example, let $(A, \mathfrak{m})$ be a complete local ring with the discrete topology. Let $\left\{A / \mathfrak{m}^{t}\right\}$ and $\left\{A_{t}\right\}$ with $A_{t}=A$ be two inverse systems of $A$-modules with discrete topology. Then the topology of $A$ with respect to the first inverse system is the $\mathfrak{m}$-adic topology, while the topology of $A$ with respect to the second is the discrete topology. However, we shall show that the topology of $\operatorname{Hom}_{R}(F, M)$ as above is independent of the direct system $\left\{F_{t}\right\}$ with $F \cong \underset{t}{\lim _{\longrightarrow}} F_{t}$.

Theorem 2.4. Let $F$ be a flat $R$-module and $M$ a linearly compact $R$-module. Let $\left\{F_{t}\right\}_{t \in K}$ be a direct system of free $R$-modules such that $F \cong \underset{t \in K}{\lim } F_{t}$. Then we have:

(i) The linearly topologized $R$-module $\operatorname{Hom}_{R}(F, M)$ defined by $\left\{F_{t}\right\}_{t \in K}$ is linearly compact and its topology is independent of this direct system.

(ii) $\operatorname{Ext}_{R}^{i}(F, M)=0$, for all $i>0$.

Proof. (i). Clearly $\operatorname{Hom}_{R}(F, M)$ is linearly compact by Lemma 2.2 (iv), (v). Let $\left\{F_{s}^{\prime}\right\}_{s \in K^{\prime}}$ be a second direct system of free $R$-modules such that $F \cong \underset{s \in K^{\prime}}{\lim ^{\prime}} \lim F_{s}^{\prime}$. Set $L=\operatorname{Hom}_{R}(F, M)$ with the topology defined by the first direct system and 
$L^{\prime}=\operatorname{Hom}_{R}(F, M)$ with the topology defined by the second one. We have to prove that $L$ is homeomorphic to $L^{\prime}$. Set $T=\bigoplus_{t \in K} F_{t}$ and $T^{\prime}=\bigoplus_{s \in K^{\prime}} F_{s}^{\prime}$. Then $T$ and $T^{\prime}$ are free $R$-modules. Note that $F$ is a homomorphic image of $T$ and it is also a homomorphic image of $T^{\prime}$. Therefore there exists a commutative diagram

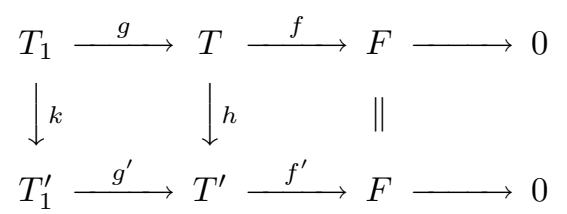

where $T_{1}$ and $T_{1}^{\prime}$ are free $R$-modules, and the rows are exact with $f, f^{\prime}$ the surjective homomorphisms and $h, k$ the homomorphisms lifting the identity map of $F$. Therefore we get the commutative diagram

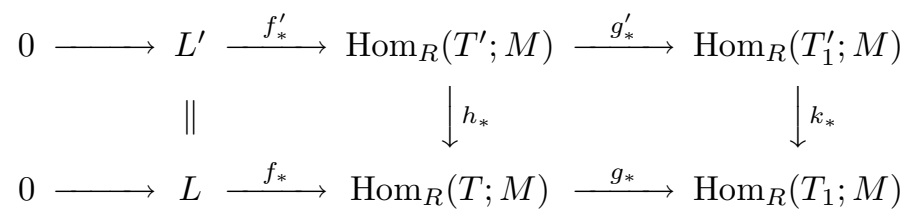

with the rows exact and the induced homomorphisms $h_{*}, k_{*}, g_{*}^{\prime}, g_{*}$ continuous. It follows that the identity map $L \longrightarrow L^{\prime}$ is continuous. Similarly, we can show that the identity map $L^{\prime} \longrightarrow L$ is continuous. Therefore $L$ is homeomorphic to $L^{\prime}$.

(ii) We obtain by [J, Theorem 4.2] a spectral sequence

$$
E_{2}^{p, q} \cong \varliminf_{t \in K}^{\lim ^{(p)}} \operatorname{Ext}_{R}^{q}\left(F_{t}, M\right) \Rightarrow \operatorname{Ext}_{R}^{i}\left(\varliminf_{t \in K}^{\left(\lim _{\longrightarrow}\right.} F_{t}, M\right) .
$$

Since $F_{t}$ is free, $E_{2}^{p, q}=0$ for $q>0$. Therefore this spectral sequence degenerates in an isomorphism

$$
{\underset{t \in K}{t \in K}}^{(i)} \operatorname{Hom}_{R}\left(F_{t}, M\right) \cong \operatorname{Ext}_{R}^{i}(F, M)
$$

Hence $\operatorname{Ext}_{R}^{i}(F, M)=0$ for all $i>0$ by Lemma 2.3.

The following immediate consequence of Theorem 2.4, which is a generalization of [M-S, Proposition 2.4], is often used in the sequel.

Corollary 2.5. Let $0 \longrightarrow M^{\prime} \longrightarrow M \longrightarrow M^{\prime \prime} \longrightarrow 0$ be a short exact sequence of linearly compact $R$-modules and $F$ a flat $R$-module. Then the derived sequence

$$
0 \longrightarrow \operatorname{Hom}_{R}\left(F, M^{\prime}\right) \longrightarrow \operatorname{Hom}_{R}(F, M) \longrightarrow \operatorname{Hom}_{R}(F, M ") \longrightarrow 0
$$

is also exact.

\section{§3. Representability of $\operatorname{Hom}_{R}(F, M)$}

In this section we need the notion of secondary representation which is due to I. G. Macdonald [M2]. This concept is in some sense dual to that of primary decomposition. An $R$-module $M$ is said to be secondary if $M \neq 0$ and for any $x \in R$, the multiplication by $x$ on $M$ is either surjective or nilpotent. The radical of the annihilator of $M$ is then a prime ideal $\mathfrak{p}$ and we say that $M$ is $\mathfrak{p}$-secondary.

Let $M$ be an $R$-module. A secondary representation of $M$ is an expression of $M$ as a finite sum $M=M_{1}+M_{2}+\ldots+M_{n}$ of $\mathfrak{p}_{i}$-secondary submodules. This representation is said to be minimal if the prime ideals $\mathfrak{p}_{i}$ are all distinct and none 
of the summands $M_{i}$ are redundant. Note that any secondary representation of $M$ can be refined to a minimal one. The set $\left\{\mathfrak{p}_{1}, \mathfrak{p}_{2}, \ldots, \mathfrak{p}_{n}\right\}$ is then independent of

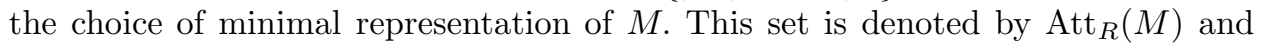
called the set of attached prime ideals of $M$. The summands $M_{i}, i=1, \ldots, n$, are called secondary component of $M$. If $M=0$ or $M$ has a secondary representation, then we say that $M$ is representable. It was shown by I. G. Macdonald [M2] and R. Y. Sharp [S] that Artinian modules and injective modules are representable.

Lemma 3.1. Let $F$ be a flat $R$-module and $M$ a linearly compact $R$-module. If $M$ is $\mathfrak{p}$-secondary, then $\operatorname{Hom}_{R}(F, M)$ is either 0 or $\mathfrak{p}$-secondary.

Proof. Suppose that $\operatorname{Hom}_{R}(F, M) \neq 0$. Given any $x \in \mathfrak{p}$, then $x^{n} M=0$ for some $n$. Therefore $x^{n} \operatorname{Hom}_{R}(F, M)=0$. Let $x \notin \mathfrak{p}$. Since $M$ is Hausdorff, 0 is a closed submodule of $M$. Because the multiplication by $x$ on $M$ is continuous, $0_{M}: x R$ is a closed submodule of $M$. Hence it is linearly compact by Lemma 2.2 (i). Therefore we have an exact sequence of linearly compact $R$-modules

$$
0 \longrightarrow 0_{M}: x R \longrightarrow M \stackrel{x}{\longrightarrow} M \longrightarrow 0 \text {. }
$$

It follows from Corollary 2.5 that the derived sequence

$$
0 \longrightarrow \operatorname{Hom}_{R}\left(F, 0_{M}: x R\right) \longrightarrow \operatorname{Hom}_{R}(F, M) \stackrel{x}{\longrightarrow} \operatorname{Hom}_{R}(F, M) \longrightarrow 0
$$

is also exact. Thus, $\operatorname{Hom}_{R}(F, M)$ is $\mathfrak{p}$-secondary.

Lemma 3.2. Let $M$ be a linearly compact $R$-module and $N$ a submodule of $M$. If $N$ is $\mathfrak{p}$-secondary, then the closure $\bar{N}$ of $N$ is also $\mathfrak{p}$-secondary.

Proof. Given any $x \in \mathfrak{p}$, then $x^{n} N=0$ for some $n$. Therefore, $0_{M}: x^{n} R \supseteq N$.

Since $0_{M}: x^{n} R$ is a closed submodule of $M$ containing $N, 0_{M}: x^{n} R \supseteq \bar{N}$. It follows that

$$
x^{n} \bar{N} \subseteq x^{n}\left(0_{M}: x^{n} R\right)=0 .
$$

Let $x \notin \mathfrak{p}$; then $x N=N$. Since $\bar{N}$ is a closed submodule of $M, x \bar{N}$ is also closed by Lemma 2.2 (ii). Moreover, because $x \bar{N} \supseteq x N=N, \quad x \bar{N} \supseteq \bar{N}$. Therefore $x \bar{N}=\bar{N}$ and hence the multiplication by $x$ on $\bar{N}$ is surjective. Thus $\bar{N}$ is $\mathfrak{p}$-secondary.

Corollary 3.3. Let $M$ be a linearly compact and representable $R$-module. Then there exists a minimal secondary representation of $M$ in which all the secondary components are linearly compact submodules.

Proof. Let $M=M_{1}+\ldots+M_{n}$ be a minimal secondary representation of $M$. Suppose that $M_{i}$ is $\mathfrak{p}_{i}$-secondary for $i=1, \ldots, n$. Then $\operatorname{Att}_{R} M=\left\{\mathfrak{p}_{1}, \ldots, \mathfrak{p}_{n}\right\}$. We use $\bar{M}_{i}$ to denote the closure of $M_{i}$ for $i=1, \ldots, n$. Then by Lemma 3.2 we get that $\bar{M}_{i}$ is $\mathfrak{p}_{i}$-secondary for $i=1, \ldots, n$. Therefore, $M=\bar{M}_{1}+\ldots+\bar{M}_{n}$ is a secondary representation of $M$. All the secondary components $\bar{M}_{i}, \quad i=1, \ldots, n$, are linearly compact by Lemma 2.2 (i). It remains to prove that this representation is minimal. If it is not minimal, then $\bar{M}_{i} \subseteq \sum_{j \neq i} \bar{M}_{j}$ for some $i$. Hence $M=\sum_{j \neq i} \bar{M}_{j}$.

It follows by Lemma 3.2 that $\mathfrak{p}_{i} \notin \operatorname{Att}_{R} M$. This gives a contradiction.

The next result can be easily derived by Corollary 3.3 and Lemma 2.2 (iii), (iv).

Corollary 3.4. Let $M$ be a linearly compact and representable $R$-module and $\mathfrak{p}$ an element in $\operatorname{Att}_{R} M$. Then there exists a homomorphic image $B$ of $M$ such that $B$ is linearly compact $\mathfrak{p}-$ secondary. 
For a representable $R$-module $M$, it is known that the isolated secondary components of $M$ depend only on $M$. By Corollary 3.3, we immediately have the following consequence.

Corollary 3.5. Let $M$ be a linearly compact and representable $R$-module. Then all the isolated secondary components of $M$ are linearly compact.

The theorem below is the main result of this section.

Theorem 3.6. Let $F$ be a flat $R$-module and $M$ a linearly compact and representable $R$-module. Then $\operatorname{Hom}_{R}(F, M)$ is linearly compact and representable.

Proof. By Corollary 3.3, we can choose a minimal secondary representation $M=$ $M_{1}+M_{2}+\ldots+M_{n}$ such that all $M_{i}, i=1, \ldots, n$, are linearly compact. By Lemma 3.1, the theorem is proved when $n=1$. If $n>1$, put $N_{1}=M_{1}$ and $N_{2}=M_{2}+\ldots+M_{n}$. Then $N_{2}$ is linearly compact by Lemma 2.2 (vi). Hence, by Lemma 2.2, $N_{1} \cap N_{2}$ and $N_{1} \oplus N_{2}$ are linearly compact. From the exact sequence of linearly compact $R$-modules

$$
0 \longrightarrow N_{1} \cap N_{2} \longrightarrow N_{1} \oplus N_{2} \longrightarrow N_{1}+N_{2} \longrightarrow 0
$$

and by Corollary 2.5 we obtain

$$
\begin{aligned}
& \operatorname{Hom}_{R}\left(F, N_{1}+N_{2}\right) \cong \operatorname{Hom}_{R}\left(F, N_{1} \oplus N_{2}\right) / \operatorname{Hom}_{R}\left(F, N_{1} \cap N_{2}\right) \\
& \left.\quad \cong \operatorname{Hom}_{R}\left(F, N_{1}\right) \oplus \operatorname{Hom}_{R}\left(F, N_{2}\right)\right) /\left(\operatorname{Hom}_{R}\left(F, N_{1}\right) \cap \operatorname{Hom}_{R}\left(F, N_{2}\right)\right) \\
& \quad \cong \operatorname{Hom}_{R}\left(F, N_{1}\right)+\operatorname{Hom}_{R}\left(F, N_{2}\right) .
\end{aligned}
$$

Therefore

$$
\operatorname{Hom}_{R}\left(F, N_{1}+N_{2}\right)=\operatorname{Hom}_{R}\left(F, N_{1}\right)+\operatorname{Hom}_{R}\left(F, N_{2}\right),
$$

where $\operatorname{Hom}_{R}\left(F, N_{1}\right)$ and $\operatorname{Hom}_{R}\left(F, N_{2}\right)$ are considered submodules of $\operatorname{Hom}_{R}(F, M)$. Now the theorem follows by induction on $n$.

Remark 3.7. As we have mentioned in $\S 1$, L. Melkersson in [Me, §5] asked "Is $\operatorname{Hom}_{R}(F, M)$ representable when $F$ is a flat $R$-module and $M$ is a representable $R$-module of finite Goldie dimension?" Theorem 3.6 is a positive answer to this question for linearly compact modules without the assumption that $M$ is of finite Goldie dimension. Recall that $M$ is said to have finite Goldie dimension if it does not contain a direct sum of infinitely many non-zero submodules. It should be noted that there exist linearly compact representable modules of infinite Goldie dimension. For instance, by [Lef], there exist linearly compact vector spaces of infinite dimension. Clearly, these spaces are representable. However, we give below an example about a large class of such modules.

Example 3.8. Let $(R, \mathfrak{m})$ be a Noetherian local $\operatorname{ring}$ with $\operatorname{dim} R>2$. Let $\mathfrak{p} \neq \mathfrak{m}$ be a prime ideal of $R$ with $h t(\mathfrak{p})>1$. Let $M$ be a linearly compact representable $R$-module which has a submodule isomorphic to the injective hull $E$ of $R / \mathfrak{m}$. Then $\operatorname{Hom}_{R}\left(R_{\mathfrak{p}}, M\right)$ is a linearly compact representable module of infinite Goldie dimension.

Proof. Note first that there always exists such a module $M$. For example, the injective hull $E$ of $R / \mathfrak{m}$ is Artinian, therefore it is representable and linearly compact with the discrect topology. Since $\operatorname{Ass}_{R_{\mathfrak{p}}}\left(\operatorname{Hom}_{R}\left(R_{\mathfrak{p}}, E\right)\right) \subseteq \operatorname{Ass}_{R_{\mathfrak{p}}}\left(\operatorname{Hom}_{R}\left(R_{\mathfrak{p}}, M\right)\right)$, it can be easily derived by $[\mathrm{M}-\mathrm{S}, 4.1]$ that $\operatorname{Ass}_{R_{\mathfrak{p}}}\left(\operatorname{Hom}_{R}\left(R_{\mathfrak{p}}, M\right)\right)=\operatorname{Spec}\left(R_{\mathfrak{p}}\right)$. Since $\operatorname{dim}_{R_{\mathfrak{p}}}\left(R_{\mathfrak{p}}\right)>1, \operatorname{Spec}\left(R_{\mathfrak{p}}\right)$ is an infinite set. Therefore $\operatorname{Ass}\left(\operatorname{Hom}_{R}\left(R_{\mathfrak{p}}, M\right)\right)$ is an 
infinite set. Hence $\operatorname{Hom}_{R}\left(R_{\mathfrak{p}}, M\right)$ is not of finite Goldie dimension. It follows from Theorem 3.6 that $\operatorname{Hom}_{R}\left(R_{\mathfrak{p}}, M\right)$ is a linearly compact representable module.

\section{$\S 4$. Co-LOCALization}

We first recall the notion of co-localization which is due to L. Melkersson and P. Schenzel $[\mathrm{M}-\mathrm{S}$. Let $M$ be an $R$-module and $S$ a multiplicative set of $R$. The co-localization of $M$ with respect to $S$ is the module $\operatorname{Hom}_{R}\left(R_{S}, M\right)$. When $M$ is Artinian, it is known by [M-S] that $\operatorname{Hom}_{R}\left(R_{S}, M\right)$ is almost never an Artinian $R_{S}-$ module. So the functor $\operatorname{Hom}_{R}\left(R_{S},-\right)$ is not closed on the category of Artinian modules, while it is always closed on the category of linearly compact representable $R$-modules by Theorem 3.6. Therefore, in some sense, it seems to the authors that it is quite interesting to study the co-localization functor on linearly compact representable $R$-modules.

Lemma 4.1. Let $S$ be a multiplicative set of $R$, and let $M$ be a linearly compact $R-$ module. Let

$$
\varphi: \operatorname{Hom}_{R}\left(R_{S}, M\right) \longrightarrow M
$$

be the homomorphism defined by $\varphi(f)=f(1)$, for any $f \in \operatorname{Hom}_{R}\left(R_{S}, M\right)$. Then

$$
\operatorname{Im} \varphi=\bigcap_{s \in S} s M
$$

Proof. Put $M^{\prime}=\bigcap_{s \in S} s M, \quad I=\bigcup_{s \in S}\left(0_{R}: s\right)$. Then $M^{\prime}$ is linearly compact by Lemma 2.2 (i), (ii). Let $R^{\prime}=R / I$. Then $M^{\prime}$ is an $R^{\prime}$-module. Let $S^{\prime}$ be the image of $S$ in $R^{\prime}$. It follows that

$$
\operatorname{Hom}_{R}\left(R_{S}, M^{\prime}\right) \cong \lim _{\longleftarrow}\left\{M_{s}^{\prime} ; g_{s}^{t}\right\},
$$

where $M_{s}^{\prime}=M^{\prime}$ for all $s \in S^{\prime}$ and $g_{s}^{t}: M_{t}^{\prime} \rightarrow M_{s}^{\prime}$ is multiplication by $a \in R^{\prime}$ if $t=$ as. We have an exact sequence of inverse systems of linearly compact $R$-modules

$$
0 \longrightarrow\left\{0_{M^{\prime}}: s, g_{s}^{t}\right\} \longrightarrow\left\{M_{s}^{\prime}, g_{s}^{t}\right\} \longrightarrow\left\{M^{\prime} / 0_{M^{\prime}}: s, g_{s}^{t}\right\} \longrightarrow 0,
$$

where the corresponding maps on $0_{M^{\prime}}: s, \quad M_{s}^{\prime}$, resp. $M^{\prime} / 0_{M^{\prime}}: s$ are multiplication by $a$ if $t=a s$. By Lemma 2.3, the homomorphism

$$
\varliminf_{\mathfrak{l}}\left\{M_{s}^{\prime}, g_{s}^{t}\right\} \longrightarrow \lim _{\mathfrak{l}}\left\{M^{\prime} / 0_{M^{\prime}}: s, g_{s}^{t}\right\}
$$

is surjective. We have by [M1, 3.12] that $s M^{\prime}=s \bigcap_{t \in S} t M=\bigcap_{t \in S} s t M=M^{\prime}$ for all $s \in S^{\prime}$. Hence, for any $t, s \in S^{\prime}$ with $t=a s$ we obtain $a M^{\prime}=M^{\prime}$. Therefore

$$
g_{s}^{t}: M^{\prime} / 0_{M^{\prime}}: t \longrightarrow M^{\prime} / 0_{M^{\prime}}: s
$$

is an isomorphism. Hence $\varliminf_{\longleftarrow}\left\{M^{\prime} / 0_{M^{\prime}}: s, g_{s}^{t}\right\} \longrightarrow M^{\prime}$ is an isomorphism. It follows that $\varphi: \operatorname{Hom}_{R}\left(R_{S}, M^{\prime}\right) \longrightarrow M^{\prime}$ is surjective.

Let $f \in \operatorname{Hom}_{R}\left(R_{S}, M\right)$. Then $f(1) \in M^{\prime}$ because $f(1)=s f(1 / s) \in s M$ for all $s \in S$. Thus $\varphi: \operatorname{Hom}_{R}\left(R_{S}, M\right) \longrightarrow M^{\prime}$ is surjective.

Now we can generalize Melkersson-Schenzel's result [M-S, Theorem 3.2] for linearly compact and representable modules as follows. 
Theorem 4.2. Let $S$ be a multiplicative set of $R$ and $M$ a linearly compact and representable $R$-module with $M=M_{1}+M_{2}+\ldots+M_{n}$ a minimal secondary representation in which all $M_{i}$ are linearly compact. Let $\mathfrak{p}_{i}=\operatorname{Rad}\left(\operatorname{Ann}_{R} M_{i}\right)$ for $i=1, \ldots, n$ and $S \cap \mathfrak{p}_{i}=\emptyset$ for $i=1, \ldots, m$, respectively $S \cap \mathfrak{p}_{i} \neq \emptyset$ for $i=$ $m+1, \ldots, n$. Then

$$
\operatorname{Hom}_{R}\left(R_{S}, M\right)=\operatorname{Hom}_{R}\left(R_{S}, M_{1}\right)+\operatorname{Hom}_{R}\left(R_{S}, M_{2}\right)+\ldots+\operatorname{Hom}_{R}\left(R_{S}, M_{m}\right)
$$

is a minimal secondary representation of $\operatorname{Hom}_{R}\left(R_{S}, M\right)$. In particular, we have

$$
\operatorname{Att}_{R}\left(\operatorname{Hom}_{R}\left(R_{S}, M\right)\right)=\left\{\mathfrak{p} \in \operatorname{Att}_{R}(M): \mathfrak{p} \cap S=\emptyset\right\} .
$$

Proof. For $i=m+1, \ldots, n$, since $S \cap \mathfrak{p}_{i} \neq \emptyset, \operatorname{Hom}_{R}\left(R_{S}, M_{i}\right)=0$. Therefore by Theorem 3.6 we have

$$
\operatorname{Hom}_{R}\left(R_{S}, M\right)=\operatorname{Hom}_{R}\left(R_{S}, M_{1}\right)+\operatorname{Hom}_{R}\left(R_{S}, M_{2}\right)+\ldots+\operatorname{Hom}_{R}\left(R_{S}, M_{m}\right) .
$$

Since $\operatorname{Hom}_{R}\left(R_{S}, M_{i}\right) \neq 0$ by Lemma 4.1 , it is $\mathfrak{p}_{i}$-secondary for $i=1, \ldots, m$ by Lemma 3.1. This representation is minimal because if it is not, then $\operatorname{Hom}_{R}\left(R_{S}, M_{i}\right)$ $\subseteq \sum_{j \neq i} \operatorname{Hom}_{R}\left(R_{S}, M_{j}\right)$ for some $i$. Therefore

$$
\varphi\left(\operatorname{Hom}_{R}\left(R_{S}, M_{i}\right)\right) \subseteq \sum_{j \neq i} \varphi\left(\operatorname{Hom}_{R}\left(R_{S}, M_{j}\right)\right)
$$

and hence $M_{i} \subseteq \sum_{j \neq i} M_{j}$ for some $i$ by Lemma 4.1. This gives a contradiction.

For an $R$-module $M$, the set

$$
\operatorname{Cos}_{R} M=\left\{\mathfrak{p} \in \operatorname{Spec} R: \operatorname{Hom}_{R}\left(R_{\mathfrak{p}}, M\right) \neq 0\right\}
$$

is called the co-support of $M$ (see [M-S]).

Corollary 4.3. Let $M$ be a linearly compact and representable $R$-module. Then we have:

(i) Any prime ideal of $R$ containing an element of $\operatorname{Att}_{R} M$ belongs to $\operatorname{Cos}_{R} M$.

(ii) Any prime ideal of $\operatorname{Cos}_{R} M$ contains an element of $\operatorname{Att}_{R} M$.

(iii) $M$ has a composition series $0=M_{0} \subset M_{1} \subset \ldots \subset M_{r-1} \subset M_{r}=M$ such that all $M_{i}, i=1, \ldots, r$, is linearly compact representable and $M_{i} / M_{i-1}$ is secondary. For such a composition series, let $\mathfrak{p}_{i}=\operatorname{Rad}\left(\operatorname{Ann}_{R}\left(M_{i} / M_{i-1}\right), i=\right.$ $1, \ldots, r$. Then we have

$$
\operatorname{Att}_{R} M \subseteq\left\{\mathfrak{p}_{1}, \ldots, \mathfrak{p}_{r}\right\} \subseteq \operatorname{Cos}_{R} M .
$$

In particular, these three sets have the same minimal elements equal to the set of minimal prime ideals containing $\operatorname{Ann}_{R} M$.

Proof. (i) and (ii) are immediate consequences of Theorem 4.2. For (iii), there exists by Corollary 3.3 a secondary representable $M=M_{1}+\ldots+M_{n}$ such that all $M_{i}$ are linearly compact. Therefore the composition series

$$
0 \subset M_{1} \subset M_{1}+M_{2} \subset \ldots \subset M_{1}+\ldots+M_{n-1} \subset M
$$

is as required. For such a composition series, it follows by Corollary 2.5 that

$$
\operatorname{Cos}_{R} M_{i}=\operatorname{Cos}_{R} M_{i-1} \cup \operatorname{Cos}_{R}\left(M_{i} / M_{i-1}\right),
$$

for all $i=1, \ldots, r$. Therefore we get $\operatorname{Att}_{R} M \subseteq\left\{\mathfrak{p}_{1}, \ldots, \mathfrak{p}_{r}\right\} \subseteq \operatorname{Cos}_{R} M$. The rest of (iii) is derived by (i), (ii) and [M2, 2.7]. 
The following result is an immediate consequence of Corollary 4.3.

Corollary 4.4. Let $M$ be a linearly compact and representable $R$-module. Then $\operatorname{Cos}_{R} M=V\left(\operatorname{Ann}_{R} M\right)$, i.e. $\operatorname{Cos}_{R} M$ is a closed subset of Spec $R$. In particular, $\operatorname{Cos} M=\emptyset$ if and only if $M=0$.

Suppose that $R$ is a Noetherian ring. Let $N$ be a finitely generated $R$-module and $M$ a linearly compact $R$-module. Let $R^{n} \stackrel{f}{\longrightarrow} R^{m} \stackrel{g}{\longrightarrow} N \longrightarrow 0$ be a finite presentation of $N$. So we get the induced exact sequence $M^{n} \stackrel{f_{*}}{\longrightarrow} M^{m} \stackrel{g_{*}}{\longrightarrow} N \otimes M$ $\longrightarrow 0$. It follows by Lemma 2.2 (iv) that $M^{n}$ and $M^{m}$ are linearly compact with respect to the product topology. Completely similar to the proof of [J] Theorem 7.4] we can show that the homomorphism $f_{*}$ is continuous. Therefore $\operatorname{Im}\left(f_{*}\right)$ is closed by Lemma 2.2 (ii). Hence $M^{m} / \operatorname{Ker}\left(g_{*}\right)$ is linearly compact by Lemma 2.2 (iii). Since $M^{m} / \operatorname{Ker}\left(g_{*}\right) \cong N \otimes M$, we can give $N \otimes M$ a topological structure deduced from the topology of $M^{m} / \operatorname{Ker}\left(g_{*}\right)$ and, with this topology, $N \otimes M$ is linearly compact.

In [M-S], L. Melkersson and P. Schenzel described the attached prime ideals of $N \otimes M$, where $R$ is a commutative ring and $M$ is an Artinian $R$-module. This is dual to a theorem of Bourbaki of $\operatorname{Ass} \operatorname{Hom}(N ; M)$ (see [B, $\S 1$, Proposition 10]). As an application of the technique of co-localization, we will describe this for the case when $R$ is a Noetherian ring and $M$ is a linearly compact representable $R$-module.

Theorem 4.5. Let $R$ be a Noetherian ring, $N$ a finitely generated $R$-module and $M$ a linearly compact $R$-module. Then we have:

(i) The topology of $N \otimes M$ defined as above does not depend on finite presentations of $N$.

(ii) Suppose in addition that $M$ is representable; then $N \otimes M$ is representable and

$$
\operatorname{Att}_{R}(N \otimes M)=\operatorname{Supp}_{R} N \cap \operatorname{Att}_{R} M .
$$

Proof. (i) Let $R^{n^{\prime}} \longrightarrow R^{m^{\prime}} \longrightarrow N \longrightarrow 0$ be the second finite presentation of $N$. Set $P=N \otimes M$ with the topology defined by the first presentation and $P^{\prime}=N \otimes M$ defined by the second one. Then, by the same method of the proof of Theorem 2.4, we can show that the identity map $P \longrightarrow P^{\prime}$ is a homeomorphism.

(ii) Clearly $V\left(\operatorname{Ann}_{R}(N \otimes M)\right) \subseteq \operatorname{Supp}_{R} N$. Since $N \otimes M$ is a homomorphic image of $M^{m}$, it is representable and

$$
\operatorname{Att}_{R}(N \otimes M) \subseteq \operatorname{Supp}_{R} N \cap \operatorname{Att}_{R} M^{m}=\operatorname{Supp}_{R} N \cap \operatorname{Att}_{R} M .
$$

Conversely, let $\mathfrak{p} \in \operatorname{Supp}_{R} N \cap \operatorname{Att}_{R} M$. Then $M$ has by Corollary 3.4 a linearly compact $\mathfrak{p}$-secondary quotient $B$. Since $R$ is Noetherian, $\mathfrak{p} B \neq B$ and $\mathfrak{p} B$ is a closed submodule of $B$ by Lemma 2.2 (vi). Therefore $C=B / \mathfrak{p} B$ is a linearly compact $\mathfrak{p}$-secondary homomorphic image of $M$ with $\mathfrak{p}=\operatorname{Ann}_{R} C$. Hence $\mathfrak{p} R_{\mathfrak{p}} \operatorname{Hom}_{R}\left(R_{\mathfrak{p}}, C\right)=0$. Therefore, by the same way as in the proof of [M-S, Lemma 5.1] and by Corollary 2.5 we can show that

$$
\operatorname{Hom}_{R}\left(R_{\mathfrak{p}}, N \otimes_{R} C\right) \cong \operatorname{Hom}_{R}\left(R_{\mathfrak{p}}, C\right) \otimes_{R_{\mathfrak{p}}} N_{\mathfrak{p}} \cong \operatorname{Hom}_{R}\left(R_{\mathfrak{p}}, C\right) \otimes_{R_{\mathfrak{p}} / \mathfrak{p} R_{\mathfrak{p}}} N_{\mathfrak{p}} / \mathfrak{p} R_{\mathfrak{p}}
$$

Since $\mathfrak{p}=\operatorname{Ann}_{R} C$, it follows by Lemma 4.4 that $\operatorname{Hom}_{R}\left(R_{\mathfrak{p}}, C\right) \neq 0$. Hence $\operatorname{Hom}_{R}\left(R_{\mathfrak{p}}, C\right)$ and $N_{\mathfrak{p}} / \mathfrak{p} R_{\mathfrak{p}}$ are non-zero vector spaces over the field $R_{\mathfrak{p}} / \mathfrak{p} R_{\mathfrak{p}}$. Therefore $\operatorname{Hom}_{R}\left(R_{\mathfrak{p}}, N \otimes_{R} C\right) \neq 0$. Hence $N \otimes C \neq 0$, so it is $\mathfrak{p}$-secondary. Therefore $\mathfrak{p} \in \operatorname{Att}_{R}(N \otimes M)$. 


\section{REFERENCES}

[B] N. Bourbaki, Algébre commutative, Hermann, Paris, 1961. MR 30:2027

[C-N] N. T. Cuong and T. T. Nam, A local homology theory for linearly compact modules, Preprint.

[J] C. U. Jensen, Les Foncteurs Dérivés de lim et leurs Applications en Théorie des Modules, Springer-Verlag, Berlin-Heidelberg-New York, 1972. MR 53:10874

[L] D. Lazard, Autour de la platitude, Bull. Soc. Math. France 97 (1969), 81-128. MR 40:7310

[Lef] S. Lefschetz, Algebraic Topology, vol. 27, Colloq. Lect. Amer. Soc., 1942. MR 4:84f

[Lep1] H. Leptin, Linear kompakte Moduln und Ringe - I, Math. Z. 62 (1955), 241-267. MR 16:1085a

[Lep2] H. Leptin, Linear kompakte Moduln und Ringe - II, Math. Z. 66 (1957), 289-327. MR 19:245a

[M1] I. G. Macdonald, Duality over complete local rings, Topology 1 (1962), 213-235. MR 27:1476

[M2] I. G. Macdonald, Secondary representation of modules over a commutative ring, Symposia Mathematica 11 (1973), 23-43. MR 49:7252

[Ma] H. Matsumura, Commutative ring theory, Cambridge University Press, 1986. MR 88h:13001

[Me] L. Melkersson, Cohomological properties of modules with secondary representations, Math. Scand. (2) 77 (1995), 197-208. MR 97d:13014

[M-S] L. Melkersson and P. Schenzel, The co-localization of an Artinian module, Proc. Edinburgh Math. Soc. 38 (1995), 121-131. MR 96a:13020

[S] R. Y. Sharp, Secondary representations for injective modules over commutative Noetherian rings, Proc. Edinburgh Math. Soc. 20 (1976), 143-151. MR 54:2639

[Z] D. Zelinsky, Linearly compact modules and rings, Amer. J. Math. 75 (1953), 79-90. MR 14:532a

Institute of Mathematics, P.O. Box 631, Boho, 10.000 Hanoi, Vietnam

E-mail address: Cuongnt@hn.vnn.vn

Institute of Mathematics, P.O. Box 631, Boho, 10.000 Hanoi, Vietnam 